\title{
Optimization of a Collapsed Mode CMUT Receiver for Maximum Off-Resonance Sensitivity
}

\author{
Mansoor Khan ${ }^{\circledR}$, Talha M. Khan, Akif Sinan Taşdelen, Mehmet Yilmaz, Abdullah Atalar ${ }^{\circledR}$, Fellow, IEEE, \\ and Hayrettin Köymen ${ }^{\circledR}$, Senior Member, IEEE
}

\begin{abstract}
We propose an airborne collapse capacitive micromachined ultrasonic transducer (CMUT) as a practical viable ultrasound transducer capable of providing a stable performance at the off-resonance frequencies. Traditional practice is to bias the CMUT plate close to collapse voltage to achieve high coupling coefficient and sense the incoming ultrasound as an open-circuit receive voltage signal of the transducer or shortcircuit receive current (SCRC). Maintaining CMUT plate in the vicinity of collapse threshold is rather difficult. In this paper, an analytic approach to design an airborne collapsedmode CMUT for maximum off-resonance sensitivity is presented. We use small-signal circuit model to evaluate the performance of a collapsed CMUT for varying operating conditions. CMUT operational parameters that yield the highest off-resonance SCRC are directly obtained from performance design curves. Collapsed CMUT plate is then biased in a critical biasing region that produces a stable and maximum off-resonance sensitivity. We experimentally verify and measure a stable sensitivity of a fabricated collapsed CMUT cell of $-60 \mathrm{~dB} V / P a$ at $100 \mathrm{kHz}$ when biased between 50 to $65 \mathrm{~V}$. We characterize our linear circuit model performance against the measured performance of collapsed CMUT in air within 4-dB tolerance.

[2018-0058]
\end{abstract}

Index Terms-CMUT, collapsed CMUT sensitivity, critical biasing region, off-resonance, small-signal circuit model.

\section{INTRODUCTION}

$\mathbf{O}$ WING to standard silicon integrated circuit (IC) fabrication technology and potential for integration with electronics, CMUTs have proved to be a viable technology in medical ultrasound. For example, the first pulse-echo 128-element, 1-D linear CMUT array was fabricated using simple photolithography process and was characterized with a wider bandwidth and higher sensitivity than piezoelectric ceramics in 2001 [1]. Commercial scanners using 1-D CMUT arrays are also reported to have produced clinical-quality

Manuscript received March 12, 2018; revised July 13, 2018; accepted July 15,2018 . This work was supported by the Scientific and Technological Research Council of Turkey under Project Grant 114E588. The work of A. Atalar was supported by the Turkish Academy of Sciences. (Corresponding author: Mansoor Khan.)

M. Khan, A. Atalar, and H. Köymen are with the Electrical and Electronics Engineering Department, Bilkent University, 06800 Ankara, Turkey (e-mail: mansoor@ee.bilkent.edu.tr).

T. M. Khan is with the Institute of Materials Science and Nanotechnology, Bilkent University, 06800 Ankara, Turkey.

A. S. Taşdelen is with the Bilkent University Acoustic and Underwater Technologies Research Center, Bilkent University, 06800 Ankara, Turkey.

M. Yilmaz is with the National Nanotechnology Research Center, Bilkent University, Ankara 06800, Turkey.

Color versions of one or more of the figures in this paper are available online at http://ieeexplore.ieee.org.

Digital Object Identifier 10.1109/JMEMS.2018.2857444 images [2], [3]. 2-D CMUT arrays with $128 \times 128$ elements have also been successfully fabricated and characterized [4]. These 2-D arrays can be integrated with electronics in the form of a 3-D multichip module by flip-chip bonding [5] . Recently [6], tuning of the center frequency of collapsedmode CMUT is investigated for inter-cardiac echo imaging. The center frequency of a collapsed CMUT is tuned between 8.7 MHz and $15.3 \mathrm{MHz}$ by varying the DC bias. Maximum transmit sensitivity of $52 \mathrm{kPa} / \mathrm{V}$ is achieved at the center frequency of $9 \mathrm{MHz}$.

In this paper we demonstrate both analytically and experimentally that an airborne CMUT in collapsed mode could be optimized and used for the detection of ultrasound more efficiently and in a stable manner than that in conventional mode. The electric field sustained between the biased collapsed plate and the substrate is larger than an un-collapsed plate owing to small insulation layer at the contact region. This small separation increases the capacitance, resulting in an improved electromechanical transformer ratio [7], [8]. This makes collapsed-mode CMUT operation a viable choice for higher acoustic output [9] with lower bandwidth than conventional CMUTs [10]. Many studies have developed and used accurate FEM models to show superior power transmission and efficiency for a collapsed mode CMUT [11].

We derive and use linear equivalent circuit model to evaluate the performance of a collapsed CMUT. SCRC normalized to incident pressure is derived from the model in terms of its lumped circuit elements. We compare SCRC with open circuit receive voltage (OCRV) and show that SCRC performance is not impaired due to electrical losses. The normalized SCRC is then optimized with bias for varying CMUT operational parameters.

\section{Analysis of Collapsed CMUT}

A cross-sectional view of collapsed CMUT plate is shown in Fig. 1 where $a$ is the clamped circular plate radius, $b$ is the contact radius, $t_{g}$ is the gap height, $t_{i}$ is the insulation layer thickness and $t_{m}$ is the plate thickness.

A static analysis of CMUT can be done using the normalized form of the Timoshenko's equation [12], [13]. We summarize this method in Appendix A for completeness.

\section{LineAr SMALL-Signal Model}

A collapsed-mode CMUT receiver, under small signal conditions can be represented by a linear equivalent-circuit-model, shown in Fig. 2. The small signal circuit parameters are 


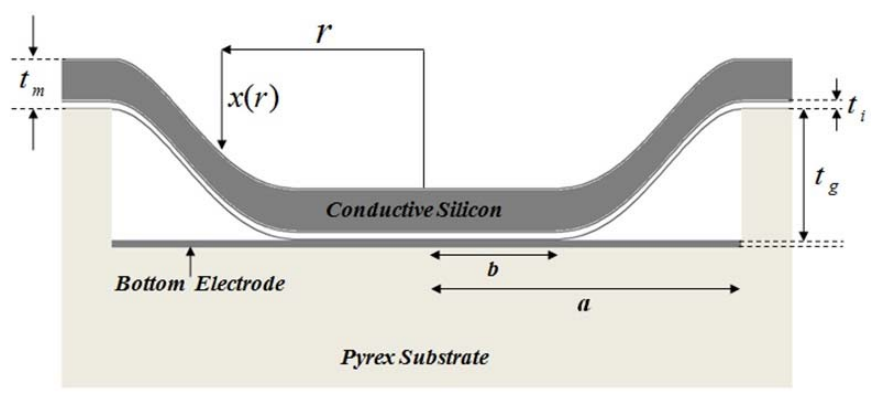

Fig. 1. Cross-sectional view of a collapsed CMUT cell.

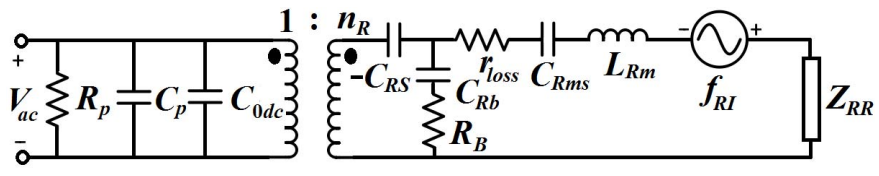

Fig. 2. Small-signal model of collapsed mode CMUT with both mechanical and electrical losses.

TABLE I

Circuit Parameters of Small Signal Model

\begin{tabular}{ll}
\hline$C_{0 d c}$ & CMUT Electrical Capacitance \\
$n_{R}$ & Electromechanical Turns Ratio \\
$C_{R S}$ & Spring Softening Capacitance \\
$C_{R m s}$ & Series Compliance Capacitance \\
$L_{R m}$ & Mass of Plate \\
$f_{R I}$ & Incident Dynamic Force \\
$Z_{R R}$ & Self Radiation Impedance \\
$C_{R b}$ & Backing Compliance \\
$R_{B}$ & Backing Loss Resistance \\
$r_{l o s s}$ & Plate Fricitional Loss Resistance \\
$C_{p}$ & Parasitic Capacitance \\
$R_{p}$ & Die-electric Loss Resistance \\
\hline
\end{tabular}

derived by linearizing the transduction and plate restoring force at the static operating point. These parameters are described in Table I.

The receive CMUT signal is the ac voltage, $V_{a c}$ at the electrical port and resulting plate rms displacement is $x_{r} \cdot{ }^{1}$ The electrical terminal voltage, $V$ and resulting total $r m s$ plate displacement $x_{R}$ is expressed as:

$$
\begin{aligned}
& V^{2}=\left[V_{D C}+V_{a c}\right]^{2} \approx V_{D C}^{2}+2 V_{D C} V_{a c} \\
& x_{R}=X_{R}+x_{r} \text { since }\left|V_{a c}\right| \ll V_{D C} \text { and }\left|x_{r}\right| \ll X_{R}
\end{aligned}
$$

$f_{R}$ is the electrical transduction force derived from the instantaneous energy, $E$, due to charge accumulated on the CMUT electrode in presence of electrical terminal voltage, $V$ :

$$
f_{R}=\frac{d E}{d x_{R}}=\frac{d}{d x_{R}}\left(\frac{1}{2} C V^{2}\right)=\frac{C_{0} V^{2}}{2 t_{g e}} g_{c}^{\prime}\left(\frac{x_{R}}{t_{g e}}\right)
$$

where $C_{0}$ is the clamped CMUT capacitance and $g_{c}($.$) is the$ capacitance polynomial defined in Appendix B. $t_{g e}=t_{g}+$ $t_{i} / \epsilon_{r}$ is the effective gap height, $\epsilon_{r}$ is the relative permittivity

\footnotetext{
${ }^{1} r m s$ displacement is defined as $x_{r}=\sqrt{\frac{1}{\pi a^{2}} \int_{0}^{a} 2 \pi x^{2}(r) r d r}$.
}

of insulation layer. $C$ is the non-linear electrical capacitance given by,

$$
\begin{aligned}
C & =C_{0} g_{c}\left(\frac{x_{R}}{t_{g e}}\right) \\
C_{0} & =\epsilon_{0} \frac{\pi a^{2}}{t_{g e}}
\end{aligned}
$$

Using (1) in (3) we linearize the transduction force, $f_{R}$ around static $X_{R}$ as:

$$
\begin{aligned}
f_{R} & =F_{R}+f_{r} \\
& =\frac{C_{0}\left(V_{D C}^{2}+2 V_{D C} V_{a c}\right)}{2 t_{g e}}\left[g_{c}^{\prime}\left(\frac{X_{R}}{t_{g e}}\right)+\frac{x_{r}}{t_{g e}} g_{c}^{\prime \prime}\left(\frac{X_{R}}{t_{g e}}\right)\right]
\end{aligned}
$$

Ignoring the higher order terms, and the DC force $F_{R}$, we write our linear transduction force $f_{r}$ in terms of normalized rms plate displacement $X_{R} / t_{g e}$ as:

$$
\begin{aligned}
f_{r} & =\frac{C_{0} V_{D C} V_{a c}}{t_{g e}} g_{c}^{\prime}\left(\frac{X_{R}}{t_{g e}}\right)+\frac{x_{r}}{t_{g e}} \frac{C_{0} V_{D C}^{2}}{2 t_{g e}} g_{c}^{\prime \prime}\left(\frac{X_{R}}{t_{g e}}\right) \\
& =n_{R} V_{a c}+\frac{x_{r}}{C_{R S}}
\end{aligned}
$$

where

$$
n_{R}=\frac{C_{0} V_{D C}}{t_{g e}} g_{c}^{\prime}\left(\frac{X_{R}}{t_{g e}}\right) \text { and } C_{R S}=\frac{2 t_{g e}^{2}}{C_{0} V_{D C}^{2} g_{c}^{\prime \prime}\left(\frac{X_{R}}{t_{g e}}\right)}
$$

$n_{R}$ is the electromechanical turns ratio at the static operating point, and $C_{R S}$ is the spring softening capacitance due to applied $V_{D C}$ whose effect opposes the plate restoring force. In terms of normalized quantities (8) can be expressed as:

$$
\begin{aligned}
n_{R}^{2} & =\frac{4}{15} \frac{C_{0}}{C_{R m 0}}\left[\frac{V_{D C}}{V_{r}} g_{c}^{\prime}\left(\frac{X_{R}}{t_{g e}}\right)\right]^{2} \\
C_{R S} & =C_{R m 0}\left[\frac{2}{15} \frac{V_{D C}^{2}}{V_{r}^{2}} g_{c}^{\prime \prime}\left(\frac{X_{R}}{t_{g e}}\right)\right]^{-1}
\end{aligned}
$$

In (9) and (10) $C_{R m 0}$ is the linear spring compliance of an un-collapsed plate:

$$
C_{R m 0}=\frac{9\left(1-\sigma^{2}\right) a^{2}}{80 \pi Y_{0} t_{m}^{3}}
$$

and $V_{r}$ is the bias required to collapse the plate in vacuum given by (31). In collapsed mode, the restoring force has a non-linear dependence on the plate displacement, therefore the restoring force is also linearized around $X_{R}$ :

$$
f_{R}+F_{R b}=\frac{x_{R}}{C_{R m}\left(x_{R}\right)}
$$

where $F_{R b}=(\sqrt{5} / 3) \pi a^{2} P_{b}$ is the uniform $r m s$ force due to ambient pressure $P_{b}$ [14]. Ignoring the higher order terms, we can write the linearized compliance at $X_{R}$ as:

$$
\begin{aligned}
f_{R}+F_{R b} & =\frac{X_{R}}{C_{R m}}+x_{r}\left[\left.\frac{d\left(x_{R} / C_{R m}\left(x_{R}\right)\right)}{d x_{R}}\right|_{x_{R}=X_{R}}\right] \\
& =\frac{X_{R}}{C_{R m}}+x_{r}\left(\frac{1}{C_{R m}}-\left.\frac{X_{R}}{C_{R m}^{2}} \frac{d C_{R m}\left(x_{R}\right)}{d x_{R}}\right|_{x_{R}=X_{R}}\right)
\end{aligned}
$$




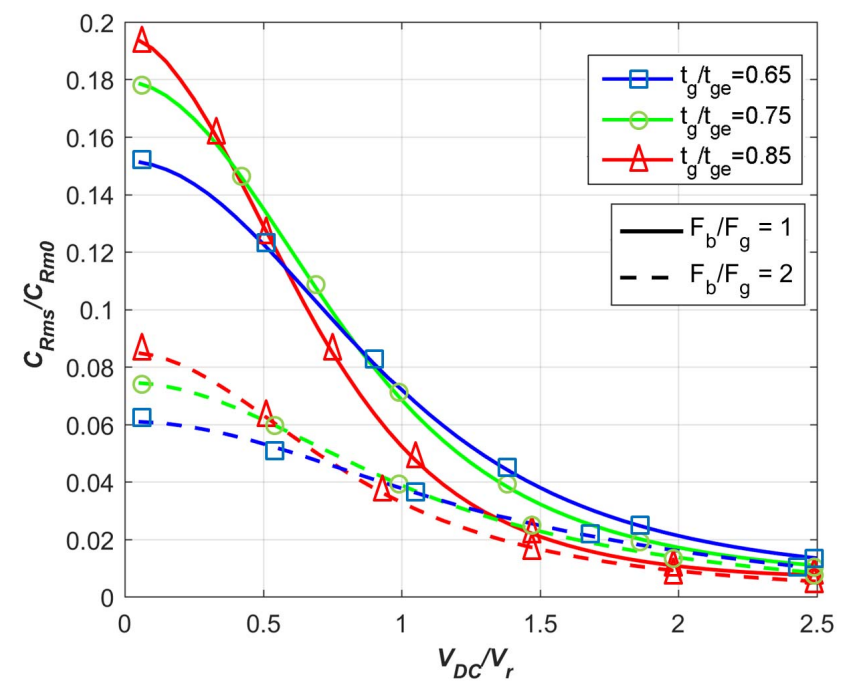

Fig. 3. Normalized series compliance, $C_{R m s} / C_{R m 0}$ as a function of normalized bias voltage, $V_{D C} / V_{r}$ for varying normalized gap heights, $t_{g} / t_{g e}$ and normalized force, $F_{b} / F_{g}$ of 1 and 2 .

In (13) $C_{R m}$ is defined as:

$$
C_{R m}=C_{R m 0} h_{c}\left(\frac{X_{R}}{t_{g e}}\right)
$$

$h_{c}($.$) is the plate compliance polynomial in X_{R} / t_{g e}$. Coefficients for capacitance and compliance polynomials are tabulated in Appendix B. From (13) we obtain our small signal compliance capacitance, $C_{R m s}$ as:

$$
C_{R m s}=C_{R m 0}\left[\frac{h_{c}^{2}\left(\frac{X_{R}}{t_{g e}}\right)}{h_{c}\left(\frac{X_{R}}{t_{g e}}\right)-\frac{X_{R}}{t_{g e}} h_{c}^{\prime}\left(\frac{X_{R}}{t_{g e}}\right)}\right]
$$

In Fig. 3, the normalized series compliance capacitance, $C_{R m s} / C_{R m 0}$ is plotted for varying normalized gap heights, $t_{g} / t_{g e}$ and normalized force, $F_{b} / F_{g}$ of 1 and 2 . For $F_{b} / F_{g}=2$ case, the plate makes large initial contact with the substrate and therefore is less compliant. As $V_{D C} / V_{r}$ increases, plate stiffens even more with increasing contact making it less compliant.

The inductance is equal to the mass of plate in rms model:

$$
L_{R m}=\pi a^{2} t_{m} \rho
$$

The small signal model is finally terminated with the collapsed CMUT radiation impedance of acoustic medium [15] as:

$$
Z_{R R}=\pi a^{2} \rho_{0} c_{0}\{R(k a, k b)+j X(k a, k b)\}
$$

where $R(k a, k b)$ and $X(k a, k b)$ are the normalized radiation resistance and reactance of collapsed-CMUT transducer, $k$ is the wavenumber, $c_{o}$ is the speed of sound and $\rho_{o}$ is the density, all specified in the immersion medium.

On the electrical side the small signal capacitance, $C_{0 d c}$, is defined at the static $X_{R} / t_{g e}$ as [16]:

$$
C_{0 d c}=C_{0} g_{c}\left(\frac{X_{R}}{t_{g e}}\right)
$$

Fig. 4 plots $g_{c}($.$) as normalized CMUT electrical capacitance$ of (18), $C_{0 d c} / C_{0}$ versus $X_{R} / t_{g e}$ for varying normalized gap heights, $t_{g} / t_{g e}$ and normalized force, $F_{b} / F_{g}$ of 1 . For large

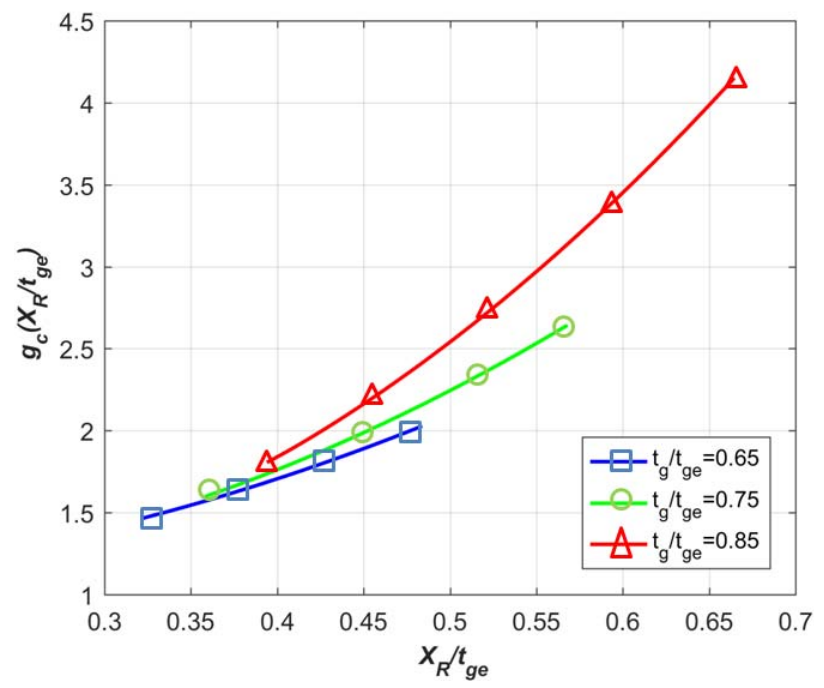

Fig. 4. CMUT capacitance polynomial, $g_{c}\left(X_{R} / t_{g e}\right)$ as a function of $X_{R} / t_{g e}$ for varying normalized gap heights, $t_{g} / t_{g e}$ and normalized force $F_{b} / F_{g}=1$.

$t_{g} / t_{g e}$ values collapsed CMUT capacitance is higher owing to thin insulation layer gap between the electrodes. As $V_{D C}$ increases, plate contact area becomes large resulting in a higher capacitance. Calculation of CMUT capacitance from the static deflection profiles is done in Appendix B.

We add lumped elements to our model to represent both electrical and mechanical losses in the CMUT. On the electrical side, $C_{p}$ is the parasitic capacitance. Dielectric loss in the insulator can be modeled as a conductance, $G_{i}=\omega C_{i} \tan \delta$ which appears parallel to the insulation capacitance, $C_{i} \cdot \tan \delta$ is the loss tangent of insulating material (for silicon dioxide $\tan \delta=0.001)$. This insulation dielectric loss can be approximated by a resistance $R_{p}$ described by [17, eq. (13)] as $R_{p}=\frac{1}{\omega\left(C_{p}+C_{o d c}\right) \tan \delta}$. The series resistance, $r_{\text {loss }}$, in the mechanical side accounts for the frictional loss of the collapsed plate.

The loss to substrate in the form of spherical waves into the solid half-space or in the form of surface waves in the interfaces is modeled by a parallel impedance branch [18] at the node after $-C_{R S}$ shown in Fig. 2. For a solid backing, $R_{B}$ is much higher than the plate radiation resistance in air. $C_{R b}$ is the series compliance of backing material.

\section{Collapsed CMUt Receiver Performance}

\section{A. Open Circuit Receive Voltage (OCRV) Sensitivity}

OCRV normalized to the incident pressure, $p$, is derived from the small signal equivalent circuit of collapsed CMUT of Fig. 2 as:

$$
\frac{V_{O C}}{p}=\frac{\frac{\pi a^{2} n_{R}}{j \omega\left(C_{0 d c}+C_{p}\right)}}{R_{R}+j \omega\left(L_{R m}+X_{R}\right)+\frac{1}{j \omega}\left(\frac{n_{R}^{2}}{C_{0 d c}+C_{p}}+\frac{1}{C_{R m s}}-\frac{1}{C_{R S}}\right)}
$$

At frequencies much lower than the resonance frequency, both the inductive reactance, $j \omega L_{R m}$ and the self-radiation 


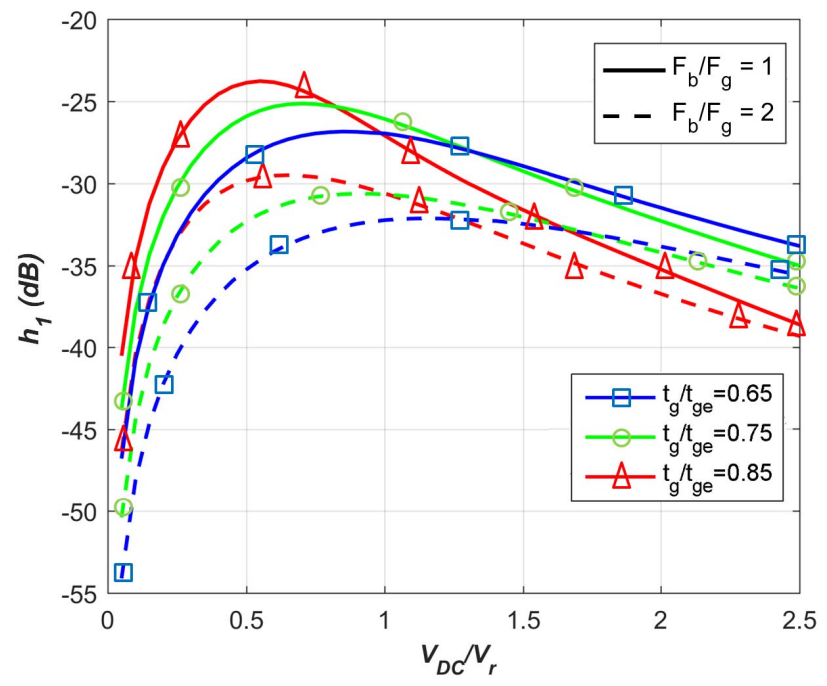

Fig. 5. Collapsed CMUT sensitivity multiplier term $h_{1}\left(\frac{X_{R}}{t_{g e}}, \frac{F_{b}}{F_{g}}, \frac{t_{g}}{t_{g e}}, \frac{C_{p}}{C_{0}}\right)$ in $\mathrm{dB}$ for $C_{p}=0$.

impedance, $R_{R}+j \omega X_{R}$ of a collapsed CMUT cell are small and can be ignored, we rewrite (19) as:

$\frac{V_{O C}}{p}=\left[\frac{3}{8} \sqrt{\frac{2\left(1-\sigma^{2}\right)}{\epsilon_{0} Y_{0}}}\left(\frac{a^{2}}{t_{m}}\right) \sqrt{\frac{t_{g e}}{t_{m}}}\right] h_{1}\left(\frac{X_{R}}{t_{g e}}, \frac{F_{b}}{F_{g}}, \frac{t_{g}}{t_{g e}}, \frac{C_{p}}{C_{0}}\right)$

with units of $\mathrm{V} / \mathrm{Pa}$. The first term of, $V_{o c} / p$, gives insight into the dimensional parameters of CMUT for highest receiver voltage sensitivity. It implies that the plate diameter must be as large as possible and it must be thin compared to both the diameter and the effective gap. The second term of (20), $h_{1}$, is given by,

$$
\begin{aligned}
& h_{1}\left(\frac{X_{R}}{t_{g e}}, \frac{F_{b}}{F_{g}}, \frac{t_{g}}{t_{g e}}, \frac{C_{p}}{C_{0}}\right) \\
& =\frac{\sqrt{\frac{3}{2}}\left(\frac{V_{D C}}{V_{r}}\right) g_{c}^{\prime}\left(\frac{X_{R}}{t_{g e}}\right)}{\left[\frac{V_{D C}}{V_{r}} g_{c}^{\prime}\left(\frac{X_{R}}{t_{g e}}\right)\right]^{2}+\frac{15}{4}\left[\frac{C_{p}}{C_{0}}+g_{c}\left(\frac{X_{R}}{t_{g e}}\right)\right]\left[\frac{1}{\overline{C_{R m s}}}-\frac{1}{\bar{C}_{R S}}\right]}
\end{aligned}
$$

$h_{1}$ of (21) is plotted in Fig. 5 for $C_{p}=0$ for various normalized gap heights and $F_{b} / F_{g}=1$ and 2 .

\section{B. Discussion}

To maximize $h_{1}$, the collapsed CMUT cell must be designed for the lowest static force, $F_{b} / F_{g}$ and the highest possible $t_{g} / t_{g e}$ with a bias equal to or slightly larger than that required for maximum sensitivity. Increasing the bias thereafter makes the plate less compliant and harder to move implying lower sensitivity. For example, when $F_{b} / F_{g}=1$, and $t_{g} / t_{g e}=0.85$, we obtain $h_{1}=-24 \mathrm{~dB}$ for $0.45<V_{D C} / V_{r}<0.65$.

We observe similar maximum sensitivity regions for higher $F_{b} / F_{g}$, however it is not prudent to choose the dimensions of CMUT for large $F_{b} / F_{g}$, as the plate becomes pre-stiffened due to large static force, resulting in more contact and

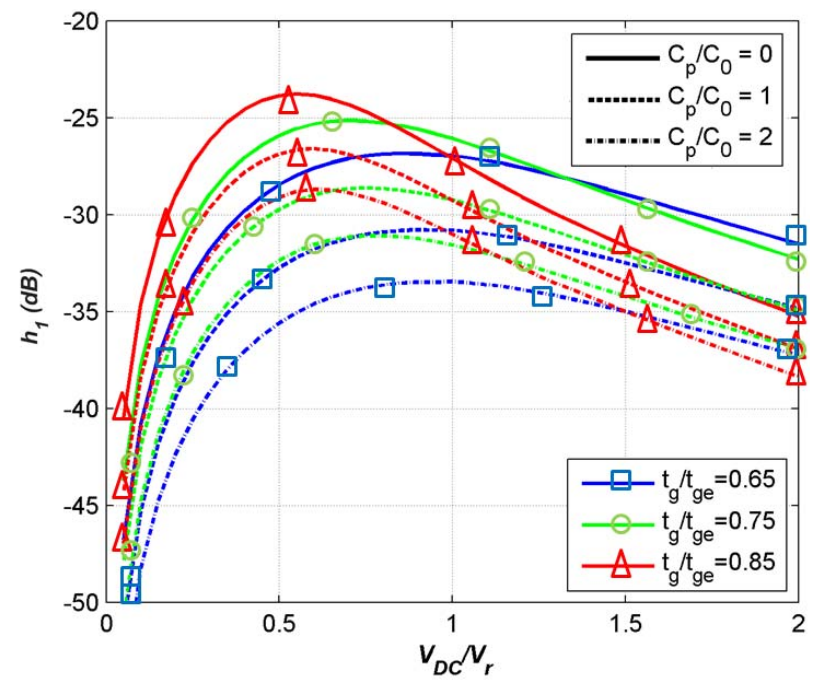

Fig. 6. Effect of relative parasitic capacitance on collapsed CMUT sensitivity multiplier term, $h_{1}$ for various normalized gap heights and $F_{b} / F_{g}=1$.

less sensitivity. Fig. 5 shows a loss of $6 \mathrm{~dB}$ for the same normalized gap height if the static normalized force, $F_{b} / F_{g}$ is doubled.

Fig. 6 demonstrates the effect of relative parasitic capacitance on sensitivity multiplier, $h_{1}$, for $F_{b} / F_{g}=1$ and various $t_{g} / t_{g e}$ values. $C_{p} / C_{0}=1$ at $t_{g} / t_{g e}=0.85$ introduces loss of $3 \mathrm{~dB}$ in the maximum sensitivity region. This loss increases to $4 \mathrm{~dB}$ if $t_{g} / t_{g e}=0.65$ is employed at $F_{b} / F_{g}=1$.

\section{Short Circuit Receive Current (SCRC) Sensitivity}

SCRC current, $i_{S C}$ (normalized to incident pressure, $p$ ) on the electrical side at off-resonance is given by,

$$
\frac{i_{s c}}{p}=\pi a^{2} \omega n_{R} \frac{C_{R m s} C_{R S}}{C_{R S}-C_{R m s}}
$$

(22) can be rewritten as:

$$
\frac{i_{s c}}{p}=\frac{\omega \pi}{5}\left[\sqrt{\frac{\epsilon_{0}\left(1-\sigma^{2}\right)}{2 Y_{0}}}\left(\frac{a^{4}}{t_{m}}\right) \frac{1}{\sqrt{t_{m} t_{g e}}}\right] h_{2}\left(\frac{X_{R}}{t_{g e}}, \frac{F_{b}}{F_{g}}, \frac{t_{g}}{t_{g e}}\right)
$$

with units of $\mathrm{A} / \mathrm{Pa}$. The first term contains CMUT geometrical parameters. For high SCRC sensitivity, CMUT must be designed with thin plate and small effective gap height, $t_{g e}$ but with larger aperture radius, $a$. The second term of (23) is given by,

$$
h_{2}\left(\frac{X_{R}}{t_{g e}}, \frac{F_{b}}{F_{g}}, \frac{t_{g}}{t_{g e}}\right)=\frac{\sqrt{\frac{3}{2}}\left(\frac{V_{D C}}{V_{r}}\right) g_{c}^{\prime}\left(\frac{X_{R}}{t_{g e}}\right)}{\left[\frac{1}{\bar{C}_{R m s}}-\frac{1}{\bar{C}_{R S}}\right]}
$$

We can use a transimpedance amplifier to measure SCRC. If we use a capacitance, $C_{f}$, as the feedback element, we get a transimpedance gain of $1 /\left(\omega C_{f}\right)$, which eliminates $\omega$ dependence of (23). Note that $h_{2}$ is independent of $C_{p}$ unlike OCRV multiplier term, $h_{1}$. Fig. 7 plots $h_{2}$ for various normalized gap heights and $F_{b} / F_{g}=1$ and 2. $h_{2}$ variation with $V_{D C} / V_{r}$ 


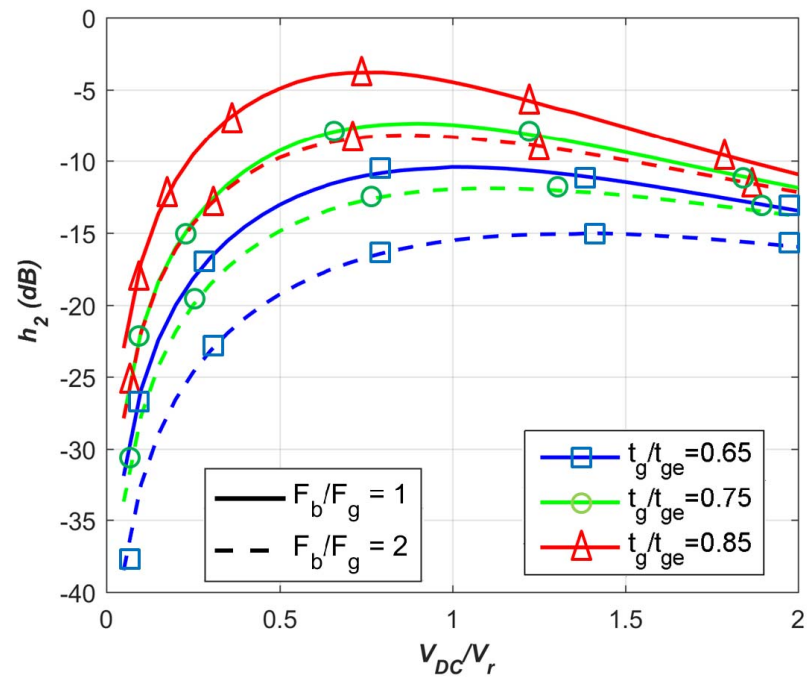

Fig. 7. Collapsed-mode CMUT SCRC sensitivity multiplier term, $h_{2}$ in $\mathrm{dB}$ for various normalized gap heights and $F_{b} / F_{g}=1$ and 2 .

TABLE II

DimENSIONAL PARAMETERS OF A FABRICATED CMUT CELL

$\begin{array}{lll}a & \text { Plate radius } & 487.6 \mu \mathrm{m} \\ t_{m} & \text { Plate thickness } & 16 \mu \mathrm{m} \\ t_{g e} & \text { Effective gap height } & 1.2 \mu \mathrm{m} \\ t_{i} & \text { Insulation layer } & 1 \mu \mathrm{m} \\ F_{b} / F_{g} & \text { Normalized static force } & 1.29 \\ t_{g} / t_{g e} & \text { Nomalized gap height } & 0.79 \\ Y_{0} & \text { Young's Modulus } & 148 \mathrm{GPa} \\ \rho_{m} & \text { Si plate density } & 2370 \mathrm{~kg} / \mathrm{m}^{3} \\ \sigma & \text { Poisson's ratio } & 0.17 \\ \epsilon_{r} & \text { Dielectric Constant } & 3.9\end{array}$

${ }^{*}$ Normalized static force is calculated at 0.9 Standard Atmospheric Pressure (SAP). $1 \mathrm{SAP}=101325 \mathrm{~Pa}$.

indicates that a collapsed-mode CMUT has a better SCRC performance than its OCRV counterpart. This is because current on electrical side is scaled by turns ratio, $n_{R}$, which increases with the bias and improves the SCRC performance.

\section{Collapsed-Mode CMUT Design for Maximum SCRC Performance}

We find collapsed CMUT physical dimensions from the operational parameters that maximize, $h_{2}$. For example for, $F_{b} / F_{g}=1$ and $t_{g} / t_{g e}=0.85$, employing $t_{i}=1 \mu \mathrm{m}$ thick silicon oxide $\left(\epsilon_{r}=3.9\right)$, with $t_{g}=1.45 \mu \mathrm{m}$ produces $t_{g e}=1.7 \mu \mathrm{m}$. A silicon plate with $t_{m}=16 \mu \mathrm{m}$ and $a=487 \mu$ m yields $F_{b} / F_{g}=1$. From (27) and silicon material properties described in Table II, the vacuum collapse voltage, $V_{r}=121.7 \mathrm{~V}$, that is $V_{D C}=92 \mathrm{~V}$ is required to obtain $h_{2}=-4 \mathrm{~dB}$. If a transimpedance amplifier is employed with a feedback capacitance, $C_{f}=1 \mathrm{pF}$ then we get a maximum transimpedance amplifier output as $-57 \mathrm{~dB} \mathrm{~V} / \mathrm{Pa}$.

\section{FABRICATION OF COLLAPSED CMUT RECEIVER}

We employ anodic wafer bonding technology similar to the one described in [19] to produce collapsed CMUTs.

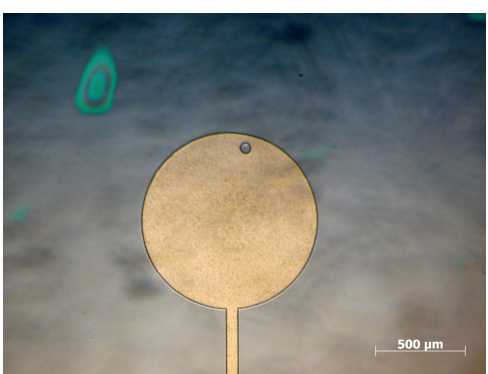

Fig. 8. Optical photo of CMUT bottom electrodes taken from the rear Pyrex side after bonding.

A (100) orientation, p-type, boron doped, wafer having $1 \mu \mathrm{m}$ top layer thermal oxide on $15 \mu \mathrm{m}$ thick device Si layer is used as a CMUT plate. A (4-inch wide, $3.3 \mathrm{~mm}$-thick) Pyrex wafer is used as the substrate. The SOI wafer has $1 \mu \mathrm{m}$ thick buried oxide (BOX) layer between the device layer and $350 \mu \mathrm{m}$ thick silicon handle layer. The total plate thickness, $t_{m}$ of Table-II has a variation of $\pm 1 \mu \mathrm{m}$ as measured by FIB/SEM.

Following the standard Pyrex wafer clean process, ${ }^{2} 35 \mathrm{~nm}$ of $\mathrm{Cr}$ is first deposited on the Pyrex wafer in an e-beam evaporation chamber. Using a standard photolithography process, circular CMUT features are UV exposed and developed. Before the Pyrex wet-etch process to carve the circular cavities, exposed $\mathrm{Cr}$ is removed by a $\mathrm{Cr}$ wet etch remover. Pyrex wafer was kept in a wet-etch (BOE 7:1) bath for 50 mins at room temperature. This produced circular CMUT cavities of $t_{g}=1.12 \mu \mathrm{m}$. CMUT cavities and electrical pads are connected by a $50 \mu \mathrm{m}$ wide channel. Bottom electrodes were deposited in an e-beam evaporation chamber and consist of $100 \mathrm{~nm} \mathrm{Ti}, 30 \mathrm{~nm} \mathrm{Pt}$ and $50 \mathrm{~nm} \mathrm{Au}$, patterned by a liftoff process. The processed Pyrex wafer containing CMUT features is then bonded with the SOI at a commercially available bonding facility. ${ }^{3}$ After bonding, the interface of the air gaps between SOI and glass substrate, are sealed with a low-viscosity epoxy resin ${ }^{4}$ which is cured in a vacuum chamber. To reveal the collapsed CMUT profiles and for subsequent electrical connections, first $350 \mu \mathrm{m}$ thick silicon handle layer is removed by $\mathrm{SF}_{6}$ based RIE process run inside an ICP chamber, while exposed gold pads are being protected by a metal shadow mask made from aluminum alloy. The BOX acts like an etch stop layer for this process, which is then removed by BOE 7:1 to reveal device silicon layer.

\section{Vi. Measurements and Model Characterization}

To validate the model predictions we use a fabricated CMUT, (see Fig. 8) parameters of which are given in Table II.

\section{A. Admittance Measurements}

The small signal model is experimentally validated by first measuring the conductance and susceptance of the fabricated CMUT in air with an impedance analyzer (HP 4194A).

\footnotetext{
${ }^{2}$ Acetone Sonication/IPA/DI water/Nitrogen blow

Device Si layer has a $0.01-100 \Omega \mathrm{cm}$ resistivity range.

${ }^{3}$ Applied Microengineering Ltd., Oxfordshire, UK.

${ }^{4}$ Biresin CR122 epoxy resin and CH122-3 hardener, Sika, Baar, Switzerland.
} 


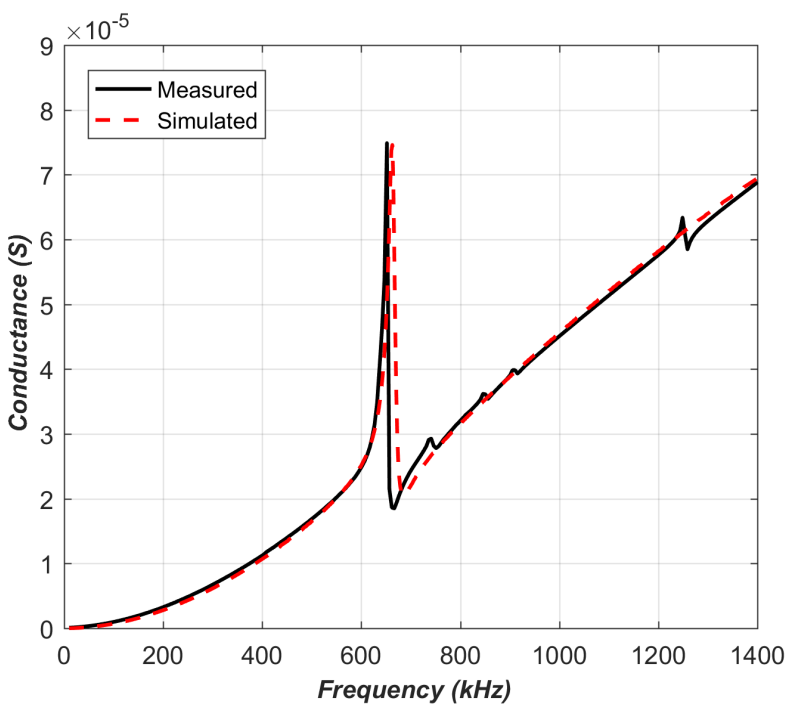

Fig. 9. Measured and simulated conductance of collapsed CMUT.
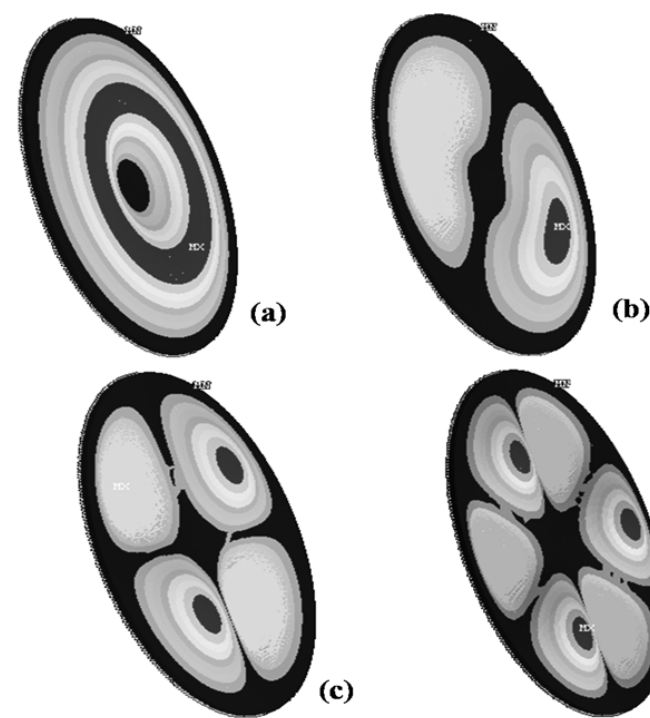

(c)

Fig. 10. Collapsed CMUT plate modes obtained in FEM modal analysis (a) first symmetrical mode at $645 \mathrm{kHz}$ (b) first asymmetrical mode at $684 \mathrm{kHz}$ (c) second asymmetrical mode at $872 \mathrm{kHz}$ (d) third asymmetrical mode at $1220 \mathrm{kHz}$.

The admittance measurements are made in long averaging mode with $20 \mathrm{~V}$ of bias voltage and $0.5 V_{p p}$ of $\mathrm{AC}$ voltage. As seen in Fig. 9 measured fundamental resonance is at $650 \mathrm{kHz}$, and the quality factor is 68 .

Fig.10 shows the lowest order symmetrical and asymmetrical modes of the collapsed CMUT plate as obtained from FEM simulations..$^{5}$ The lowest order symmetrical mode (Fig. 10(a)) occurs at $645 \mathrm{kHz}$ as verified by the experiment. Asymmetrical modes of the plate are also excited due to small asymmetries arising from production inaccuracies. These resonances are also visible in the measurement results as small peaks.

${ }^{5}$ FEM simulations are carried out in Ansys R14.5 (Ansys Inc., Canonsburg, PA). Modal analysis of a 3D collapsed CMUT cell is done and lowest plate symmetrical and asymmetrical modes are extracted.

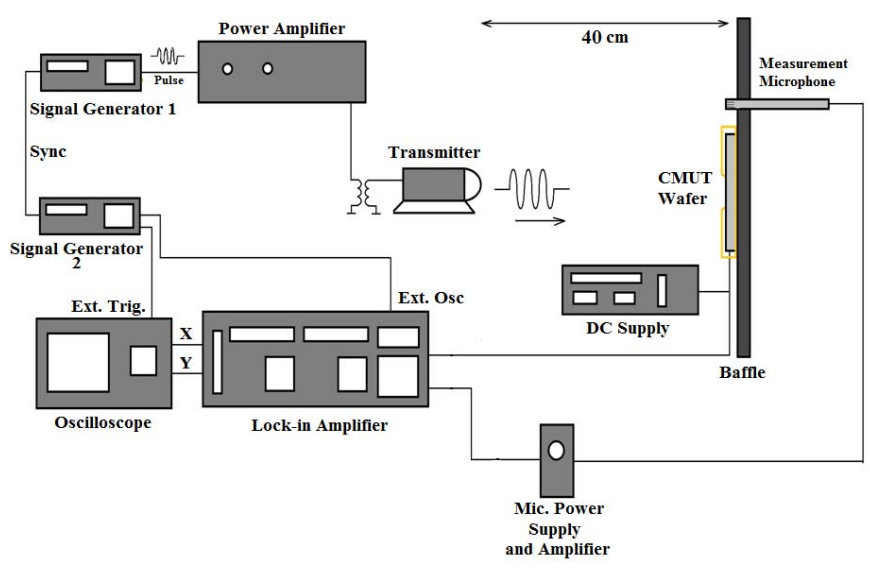

Fig. 11. Pulse measurement setup with lock-in amplifier.

The radiation resistance of spherical waves propagating in isotropic solids is derived by Blake, Jr., [20]. Baseline of the measured conductance is reproduced by $R_{B}$ which is 10 times larger than plate radiation resistance in air with a series loss of $5.6 S \rho_{0} c_{o}$. This series loss (due to friction of collapsed plate) together with the backing loss, lowers the overall SNR of the received signal. We measure $C_{p}$ as 0.98 times $C_{0}$.

To match the measured resonance, we need to set $t_{m}=$ $15.4 \mu \mathrm{m}$ and $a=492 \mu \mathrm{m}$. The peak conductance is adjusted by setting $t_{g e}=1.53 \mu \mathrm{m}$ which does not result in a significant shift in the resonance frequency.

\section{B. Receive Sensitivity Performance Measurements}

SCRC sensitivity of CMUT is measured at low ultrasound frequency of $100 \mathrm{kHz}$ using the setup shown in Fig. 11. First, the CMUT wafer is covered with a metal shield to reduce the coupling. A low noise OPAMP (MAX4475) ${ }^{6}$ in a shield is placed right next to CMUT with $R_{f}=1 \mathrm{G} \Omega$ and $C_{f}=1 \mathrm{pF}$ as its parallel feedback impedance. CMUT is connected directly to the inverting input while the noninverting input of the OPAMP is connected to the ground. CMUT bias voltage is applied to the bottom electrode. The output voltage of amplifier, $\left(i_{s c} / \omega C_{f}\right)$ is recorded with varying bias at each frequency. The small-signal model of Fig. 2 is simulated in a circuit simulator ${ }^{7}$ using the employed OPAMP at off-resonance, to include the pre-amplifier gain in SCRC as shown in Fig. 12c.

\section{Time Domain Pulse Measurements}

Receiver CMUT is mounted $40 \mathrm{~cm}$ away on a planar wooden hardboard baffle, and is insonified by a wideband airborne transmitter $^{8}$ which is driven by a signal generator with a $3 V_{p}$ pulse of $1 \mathrm{~ms}$ duration (100 cycles at $100 \mathrm{kHz}$ ). A gain of 75 is provided by an amplifier. ${ }^{9}$

\footnotetext{
${ }^{6}$ Maxim Integrated 160 Rio Robles, San Jose, CA 95134 USA, www.maximintegrated.com

${ }^{7}$ Advanced Design Systems, Keysight Technologies, Santa Rosa, CA.

${ }^{8}$ Series 600 Instrument Grade, Ultrasonic Transmitter, SensComp Inc. Livonia, USA

${ }^{9}$ Krohn-Hite 7500, Krohn-Hite Corporation, Brockton, MA
} 


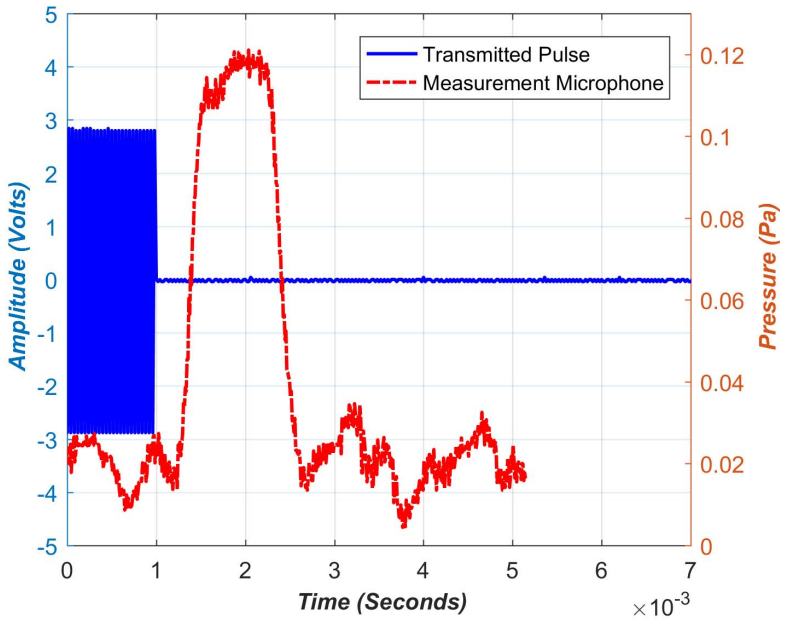

(a)

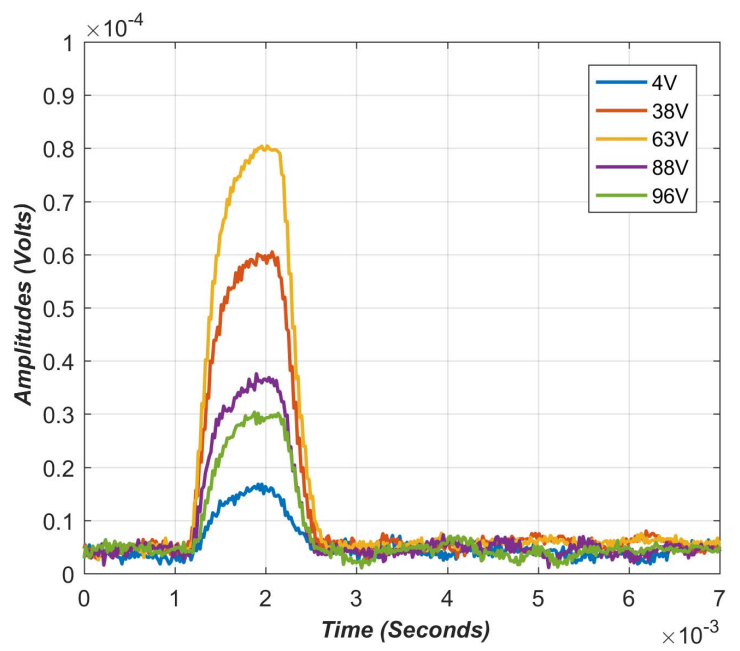

(b)

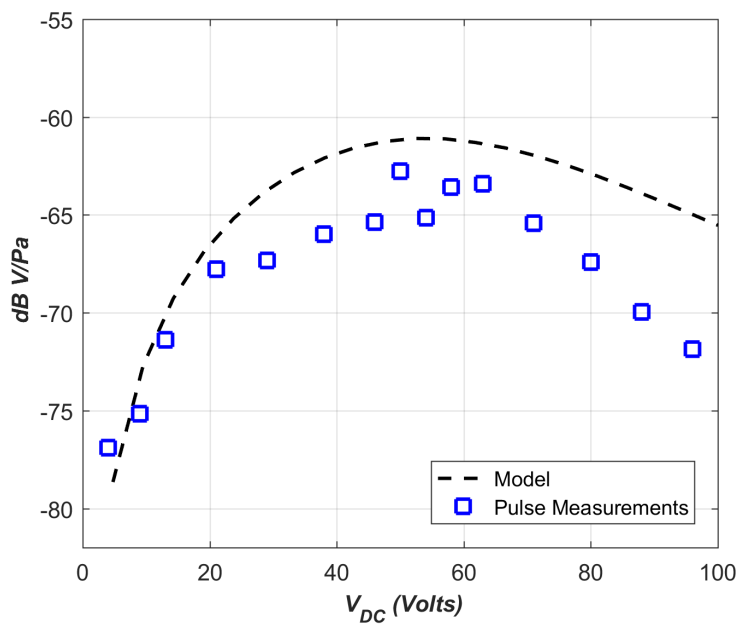

(c)

Fig. 12. Pulse Measurements at $100 \mathrm{kHz}$ (a) transmitted pulse and measurement microphone recorded pressure (b) received signal envelopes for varying DC bias is recorded on an oscilloscope (c) squares in the figure shows the recorded sensitivity at $100 \mathrm{kHz}$ of CMUT at preamplifier output in $\mathrm{dB} \mathrm{V} / \mathrm{Pa}$ from the time domain pulse data.

Pulse repetition rate is kept $200 \mathrm{~ms}$, to avoid any interference due to reflections or incoming transmitting pulse (See Fig.12a). A lock-in amplifier (Stanford Research Systems,

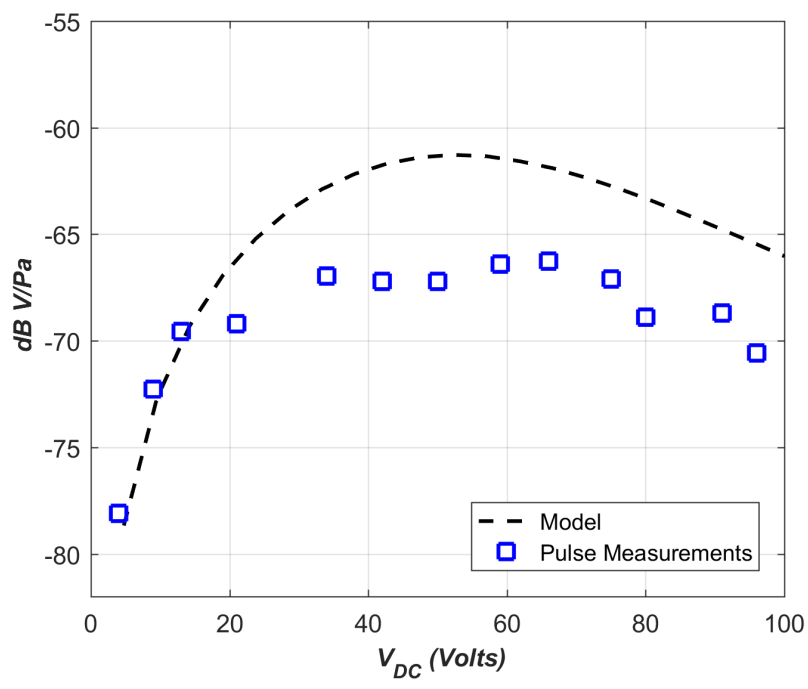

Fig. 13. Variation of measured CMUT sensitivity against model at $110 \mathrm{kHz}$.

SRS 830) with a set time constant of $100 \mu$ s and filter roll off of $12 \mathrm{~dB} /$ octave is employed to measure the amplitudes of receive signal envelopes on an oscilloscope. The incident acoustic pressure is first measured by a calibrated pressure microphone (pressure-field microphone, B\&K 4138) mounted on a preamplifier (B\&K 2633) using an adaptor (B\&K UA 160). The microphone is polarized by a power supply (B\&K Type 2807). The sensitivity of the microphone sub system is $-66.9 \mathrm{~dB} \mathrm{~V} / \mathrm{Pa}$ (or $0.452 \mathrm{mV} / \mathrm{Pa}$ ). The microphone output voltage is converted into pressure using its calibration data and referred to the second axis at the right of Fig. 12a. At the same distance of $40 \mathrm{~cm}$, we measure the baffle pressure of $0.12 \mathrm{~Pa}$. This reference pressure is used to normalize the received CMUT acoustic envelopes of Fig. $12 b$ for varying DC bias. Measured sensitivity of fabricated CMUT cell is then compared with the model prediction at $100 \mathrm{kHz}$ (Fig. 12c).

We repeat the pulse measurements at an alternate offresonance frequency of $110 \mathrm{kHz}$, with the same pulse excitation duration of $1 \mathrm{~ms}(110$ cycles at $110 \mathrm{kHz})$. The variation of measured CMUT sensitivity against model is shown in Fig. 13.

\section{Discussion}

Measured SCRC sensitivity at $100 \mathrm{kHz}$ is shown in Fig. 12. We characterize maximum receive performance of $-60 \mathrm{~dB} \mathrm{~V} / \mathrm{Pa}$ over the biasing range of 50 to 65 Volts. Measured absolute receive sensitivity of collapsed CMUT receivers is in very good agreement with what is predicted by our small signal model. We repeat the same measurements at an alternate off-resonance frequency of $110 \mathrm{kHz}$ (see Fig. 13) and obtain the same variation of CMUT performance against varying bias. The critical biasing region predicted by the model of fabricated CMUT is characterized within $4 \mathrm{~dB}$ on average at off-resonance frequencies. We record multiple receive envelopes at each bias with a variance of less than $2 \mathrm{~dB}$. Averaged envelopes are shown in this paper. Average SNR of these measurements for varying bias is 
about $21 \mathrm{~dB}$. We report absolute pressure measurements in air which are affected by the measurement environment differently at different frequencies.

\section{CONCLUSION}

This paper shows that an optimized off-resonance CMUT receiver can be more stable and reasonably sensitive in comparison to an uncollapsed CMUT receiver. A linearequivalent-circuit model based approach is employed to characterize the receive sensitivity of collapse mode CMUT receiver. We demonstrated through low frequency SCRC measurements that a fabricated collapsed CMUT cell maximum receive sensitivity can be very accurately characterized by our linear small signal model. Conductance baseline correction is first made by adding backing loss impedance in the model. After impedance characterizations, we rederive CMUT receive performance with backing loss and compare it with SCRC measurements. Variation of the measured SCRC shows that a collapsed mode CMUT receiver sensitivity can be optimized with bias and that if it is biased in the critical maximum sensitivity region a stable maximum performance can be achieved at off-resonance frequencies.

\section{APPENDIX A}

\section{Static Analysis of Collapsed Mode CMUT}

Collapsed CMUT bending profile $x(r)$ is determined by employing radial dependent electrical force in presence of uniformly distributed mechanical force (due to ambient pressure, $\left.P_{b}\right)$ in Timoshenko's formulation's [21]. To avoid a large set of collapse bending profile calculations for each different CMUT design, we start with the normalized form of Timoshenko's Eq. (3) in [12]:

$\bar{r} \frac{d}{d \bar{r}}\left(\frac{1}{\bar{r}} \frac{d}{d \bar{r}}\left(\bar{r} \frac{d x(\bar{r})}{d \bar{r}}\right)\right)=64 \int_{\bar{b}}^{\bar{r}}\left(\frac{P_{b}}{P_{g}}+\frac{2\left(V_{D C} / V_{r}\right)^{2}}{9(1-\bar{x}(\zeta))^{2}}\right) \zeta d \zeta$

where $r$ is the circular plate radial variable and the normalized variables are:

$$
\bar{r}=\frac{r}{a}, \quad \bar{b}=\frac{b}{a}, \quad \bar{x}(.)=\frac{x(.)}{t_{g e}}
$$

$P_{g}$ is the pressure required to deflect the plate by its effective gap height, $t_{g e}$ at zero bias and $V_{r}$ is the bias required to collapse the CMUT plate in vacuum.

$$
P_{g}=\frac{64 D t_{g e}}{a^{4}}, \quad V_{r}=\frac{16}{3 a^{2}} \sqrt{\frac{D t_{g e}^{3}}{\epsilon_{0}}}
$$

where $D$ is the plate flexural rigidity constant. $D=Y_{0} t_{m}^{3} /$ 12(1- $\left.\sigma^{2}\right) . Y_{0}$ and $\sigma$ are the Young's modulus and Poison ratio of the plate, respectively. (25) is solved numerically with the following boundary conditions:

$$
\begin{aligned}
\left.\bar{x}(\bar{r})\right|_{\bar{r}=1} & =0,\left.\quad \bar{x}(\bar{r})\right|_{\bar{r}=\bar{b}}=\frac{t_{g}}{t_{g e}},\left.\quad \frac{d}{d \bar{r}} \bar{x}(\bar{r})\right|_{\bar{r}=1}=0, \\
\left.\frac{d}{d \bar{r}} \bar{x}(\bar{r})\right|_{\bar{r}=\bar{b}} & =0,\left.\quad \frac{d^{2}}{d \bar{r}^{2}} \bar{x}(\bar{r})\right|_{\bar{r}=\bar{b}}=0
\end{aligned}
$$

For different combinations of CMUT operational parameters $P_{b} / P_{g}, V_{D C} / V_{r}$, and $t_{g} / t_{g e}$ resulting collapsed $r m s$ plate displacement, $X_{R}$ is calculated numerically in the MATLAB routine described in [22]. The lumped circuit elements are then uniquely defined as polynomials in $X_{R} / t_{g e}$.

\section{APPENDIX B \\ Collapsed-Mode CMUT Capacitance AND COMPLIANCE POLYNOMIALS}

$g_{c}$ (.) in (4) is a polynomial in $X_{R} / t_{g e}$ corresponding to the CMUT nonlinear capacitance

$$
g_{c}\left(\frac{X_{R}}{t_{g e}}\right)=\sum_{i=0}^{3} n_{i}\left(\frac{X_{R}}{t_{g e}}\right)^{i}
$$

For a given CMUT design first the operational parameters are determined from its physical dimensional parameters. For the obtained fixed normalized static pressure, $P_{b} / P_{g}$, and gap height, $t_{g} / t_{g e},(25)$ is solved by varying $V_{D C} / V_{r}$. This yields values for the collapsed bending profile, $\bar{x}(\bar{r})$. Corresponding nonlinear CMUT capacitance $C$ for each given deflection profile $\bar{x}(\bar{r})$ is obtained from (30) as:

$$
C=C_{0} \int_{0}^{1} \frac{2 \bar{r}}{1-\bar{x}(\bar{r})} d \bar{r}
$$

For each collapsed profile $\bar{x}(\bar{r}),(30)$ is solved numerically in the MATLAB routine of [22] and corresponding values for normalized capacitance, $C / C_{0}$ are obtained. A third degree curve fitting to $C / C_{0}$ against $X_{R} / t_{g e}$ yields polynomial coefficients $n_{i}$ of (29).

Compliance in collapsed mode of operation is a nonlinear function of normalized rms plate displacement $X_{R} / t_{g e}$ and decreases with increasing contact of plate on the substrate. We define $C_{R m}$ as the ratio of $X_{R}$ to total rms force on the membrane (including both electrical and ambient force)

$$
C_{R m}=\frac{X_{R}}{F_{R}+F_{R b}}
$$

where $F_{R}$ is the nonlinear electrical force of (6) and $F_{R b}$ is the rms static force due to ambient pressure $P_{b}$. (31) can be expressed in its normalized form as:

$$
\frac{C_{R m}}{C_{R m 0}}=\frac{\sqrt{5}\left(X_{R} / t_{g e}\right)}{\frac{2 \sqrt{5}}{15}\left(\frac{V_{D C}}{V_{r}}\right)^{2} \frac{d C}{d \overline{X_{R}}}+\frac{P_{b}}{P_{g}}}
$$

With $C / C_{0}$ data in hand, $d C / d \bar{X}_{R}$ is obtained from the first derivative of (30) numerically for any given $P_{b} / P_{g}$, and $t_{g} / t_{g e}$. A fourth degree curve fitting to the normalized compliance curve $C_{R m} / C_{R m 0}$ yields polynomial coefficients $m_{i}$ for $h_{c}($.) of (33).

$$
h_{c}\left(\frac{X_{R}}{t_{g e}}\right)=\sum_{i=0}^{4} m_{i}\left(\frac{X_{R}}{t_{g e}}\right)^{i}
$$

For any arbitrary CMUT operating conditions once $g_{c}($.$) and$ $h_{c}\left(\right.$.) polynomials are obtained, the sensitivity multiplier $h_{2}$ of the collapsed CMUT can be calculated from (24). Short circuit receive performance can then be readily obtained from (23) for given material and physical dimensional parameters of CMUT. 
TABLE III

Polynomial Coefficients For $P_{b} / P_{g}=1.29$ And $t_{g} / t_{g e}=0.79$

\begin{tabular}{llllll}
\hline$n_{i}$ & $n_{0}$ & $n_{1}$ & $n_{2}$ & $n_{3}$ & \\
& 0.9985 & -0.7316 & 6.5870 & 0.3577 & \\
$m_{i}$ & $m_{0}$ & $m_{1}$ & $m_{2}$ & $m_{3}$ & $m_{4}$ \\
& 6.735 & -22.330 & 8.3695 & 36.1269 & -31.9043 \\
\hline
\end{tabular}

Table III contains the polynomial coefficients obtained for CMUT capacitance and compliance for normalized static pressure, $P_{b} / P_{g}$ of 1.29 and normalized gap height $t_{g} / t_{g e}$ of 0.79 .

\section{ACKNOWLEDGMENT}

We gratefully acknowledge the support and recommendations of Tony Rogers at Applied Microengineering Ltd., Oxfordshire, UK for the anodic bonding.

\section{REFERENCES}

[1] Ö. Oralkan et al., "Capacitive micromachined ultrasonic transducers: Next-generation arrays for acoustic imaging?" IEEE Trans. Ultrason., Ferroelectr, Freq. Control, vol. 49, no. 11, pp. 1596-1610, Nov. 2002.

[2] D. M. Mills and L. S. Smith, "Real-time in-vivo imaging with capacitive micromachined ultrasound transducer (CMUT) linear arrays," in Proc. IEEE Symp. Ultrason., vol. 1, Oct. 2003, pp. 568-571.

[3] D. M. Mills, "Medical imaging with capacitive micromachined ultrasound transducer (cMUT) arrays," in Proc. IEEE Symp. Ultrason., vol. 1, Aug. 2004, pp. 384-390.

[4] C. H. Cheng, E. M. Chow, X. Jin, S. Ergun, and B. T. Khuri-Yakub, "An efficient electrical addressing method using through-wafer vias for two-dimensional ultrasonic arrays," in Proc. IEEE Symp. Ultrason., vol. 2, Oct. 2000, pp. 1179-1182.

[5] J. F. Kuhmann, M. Heschel, S. Bouwstra, F. Baleras, and C. Massit, "Through wafer interconnects and flip-chip bonding: A toolbox for advanced hybrid technologies for MEMS," in Proc. 13th Eur. Conf. Solid-State Transducers, Sep. 1999, pp. 265-272.

[6] M. Pekař, W. U. Dittmer, N. Mihajlović, G. van Soest, and N. de Jong, "Frequency tuning of collapse-mode capacitive micromachined ultrasonic transducer," Ultrasonics, vol. 74, pp. 144-152, Feb. 2017.

[7] B. Bayram, E. Haeggstrom, G. G. Yaralioglu, and B. T. Khuri-Yakub, "A new regime for operating capacitive micromachined ultrasonic transducers," IEEE Trans. Ultrason., Ferroelectr., Freq. Control, vol. 50, no. 9, pp. 1184-1190, Sep. 2003

[8] Ö. Oralkan et al., "Experimental characterization of collapse-mode CMUT operation," IEEE Trans. Ultrason., Ferroelectr., Freq. Control, vol. 53, no. 8, pp. 1513-1523, Aug. 2006.

[9] S. Olcum, F. Y. Yamaner, A. Bozkurt, H. Köymen, and A. Atalar, "Deepcollapse operation of capacitive micromachined ultrasonic transducers," IEEE Trans. Ultrason., Ferroelectr., Freq. Control, vol. 58, no. 11, pp. 2475-2483, Nov. 2011.

[10] K. K. Park, Ö. Oralkan, and B. T. Khuri-Yakub, "A comparison between conventional and collapse-mode capacitive micromachined ultrasonic transducers in 10-MHz 1-D arrays," IEEE Trans. Ultrason., Ferroelectr., Freq. Control, vol. 60, no. 6, pp. 1245-1255, Jun. 2013.

[11] Y. Huang et al., "Collapsed regime operation of capacitive micromachined ultrasonic transducers based on wafer-bonding technique," in Proc. IEEE Symp. Ultrason., vol. 2, Oct. 2003, pp. 1161-1164.

[12] E. Aydoğdu, A. Ozgurluk, A. Atalar, and H. Köymen, "Parametric nonlinear lumped element model for circular CMUTs in collapsed mode," IEEE Trans. Ultrason., Ferroelectr., Freq. Control, vol. 61, no. 1, pp. 173-181, Jan. 2014.

[13] E. Aydoğdu, A. Ozgurluk, H. K. Oguz, A. Atalar, and H. Köymen, "Lumped element model of single CMUT in collapsed mode," in Proc. IEEE Int. Ultrason. Symp. (IUS), Jul. 2013, pp. 283-286.

[14] H. Köymen et al., "An improved lumped element nonlinear circuit model for a circular CMUT cell," IEEE Trans. Ultrason., Ferroelectr., Freq. Control, vol. 59, no. 8, pp. 1791-1799, Aug. 2012.

[15] A. Ozgurluk, A. Atalar, H. Köymen, and S. Olcum, "Radiation impedance of collapsed capacitive micromachined ultrasonic transducers," IEEE Trans. Ultrason., Ferroelectr., Freq. Control, vol. 59, no. 6, pp. 1301-1308, Jun. 2012.
[16] E. Aydoğdu, A. Ozgurluk, A. Atalar, and H. Köymen, "Lumped element modeling of CMUT arrays in collapsed mode," in Proc. IEEE Int. Ultrason. Symp. (IUS), Sep. 2014, pp. 309-312.

[17] A. Unlugedik, A. S. Taşdelen, A. Atalar, and H. Köymen, "Designing transmitting CMUT cells for airborne applications," IEEE Trans. Ultrason., Ferroelectr., Freq. Control, vol. 61, no. 11, pp. 1899-1910, Nov. 2014.

[18] H. Köymen, A. Atalar, and A. S. Taşdelen, "Bilateral CMUT cells and arrays: Equivalent circuits, diffraction constants and substrate impedance," IEEE Trans. Ultrason., Ferroelectr., Freq. Control, vol. 64, no. 2, pp. 414-423, Feb. 2017.

[19] M. Bellaredj, G. Bourbon, V. Walter, P. Le Moal, and M. Berthillier, "Anodic bonding using SOI wafer for fabrication of capacitive micromachined ultrasonic transducers," J. Micromech. Microeng., vol. 24, no. 2, p. $025009,2014$.

[20] F. Blake, Jr., "Spherical wave propagation in solid media," J. Acoust. Soc. Amer, vol. 24, no. 2, pp. 211-215, 1952.

[21] S. P. Timoshenko and S. Woinowsky-Krieger, Theory of Plates and Shells. New York, NY, USA: McGraw-Hill, 1959.

[22] E. Aydoğdu, "Lumped element modeling of circular CMUT in collopsed mode," Ph.D. dissertation, Dept. Elect. Electron. Eng., Bilkent Univ., Ankara, Turkey, 2014.

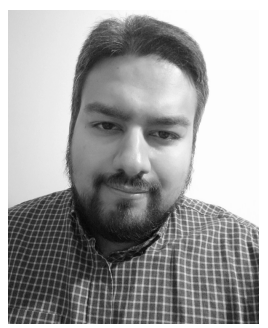

Mansoor Khan received the B.S. degree in electrical and electronics engineering from the National University of Sciences and Technology, Karachi, Pakistan, in 2007, and the M.Sc. degree in signal processing from Nanyang Technological University, Singapore, in 2009. He is currently pursuing the $\mathrm{Ph} . \mathrm{D}$. degree in electrical and electronics engineering with Bilkent University, Ankara, Turkey. Since 2013, he has been a Research and Teaching Assistant with Bilkent University. His current research interests include linear circuit modeling and characterization of collapsed CMUTs as ultrasound sensors.

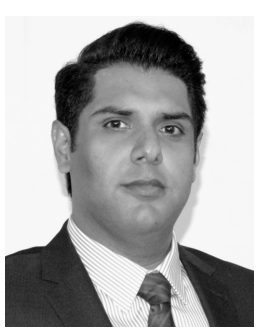

Talha M. Khan received the M.S. degree in electrical engineering from the National University of Sciences and Technology, Islamabad, Pakistan, in 2013. $\mathrm{He}$ is currently pursuing the $\mathrm{Ph} . \mathrm{D}$. degree with the National Nanotechnology Center, Bilkent University, Ankara, Turkey. He joined the Faculty of the Department of Electrical Engineering, PIET, Multan, Pakistan, as a Lecturer. His research interests include Bio-MEMS, MEMS sensors, airborne acoustics, and ultrasonic transducer design.

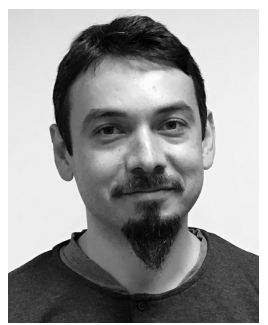

Akif Sinan Taşdelen was born in Ankara, Turkey, in 1981. He received the B.S. and M.S. degrees in electrical and electronics engineering from Bilkent University, Ankara, in 2004 and 2007, respectively. In 2008, he joined the Bilkent University Acoustics and Underwater Technologies Research Center, where he is currently a Chief Technical Research Engineer. His research interests include passive coherent location radar, underwater acoustics, transducer array design, and biomedical ultrasound. 


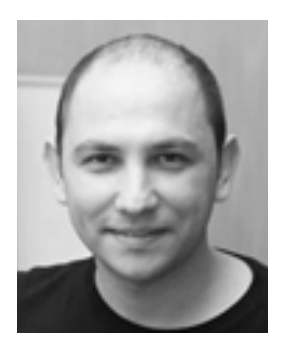

Mehmet Yilmaz received the B.S. degree (Hons.) from the Izmir Institute of Technology, the M.S. degree from Koç University, and the Ph.D. degree from Columbia University, all in mechanical engineering. During his M.S. and Ph.D. degrees, he has specialized in design and microfabrication of MEMS and integration of MEMS with nanostructures. During his Ph.D. degree, he specialized in nanomechanical characterization of materials in situ scanning electron microscopes. He joined the IBM Microelectronics Division, Albany Nanotechnology Research and Development Center, Albany, NY, USA, where he was involved in developing reactive-ion etching (RIE) processes for via patterning and developing new integration schemes for 10- and 7-nm technology nodes, and silicon 3-D integration technologies. He is currently a Principal Investigator with the National Nanotechnology Research Center, Bilkent University. In spirit, he is an academician, scientist, engineer, inventor, and entrepreneur. $\mathrm{He}$ is a Co-Inventor of two U.S. patents from the research and development efforts during his time at IBM. He is interested in mechanical characterization, elastic strain engineering, understanding, and tuning the material properties at small length scales for energy and information technology applications, and developing new unit processes and integration processes for batch-compatible nanofabricated, high yield, MEMS and NEMS devices for energy, information technology, and health applications.

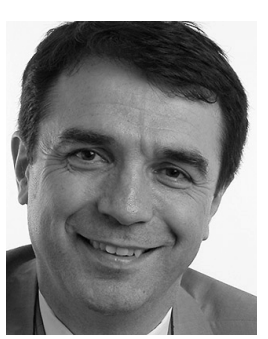

Abdullah Atalar (F'07) received the B.S. degree from Middle East Technical University, Ankara, Turkey, in 1974, and the M.S. and Ph.D. degrees from Stanford University, Stanford, CA, USA, in 1976 and 1978, respectively, all in electrical engineering. From 1978 to 1980, he was a Post-Doctoral Fellow and then an Engineering Research Associate with Stanford University. He was Hewlett Packard Labs, Palo Alto, CA, USA, for one year. From 1980 to 1986, he was an Assistant Professor with the Faculty of the Middle East Technical University. In 1983, on leave from the University, he was with Ernst Leitz Wetzlar (now Leica), Wetzlar, Germany. In 1986, he joined Bilkent University as the Chairman of the Electrical and Electronics Engineering Department and served in the founding of the Department, where he is currently a Professor. In 1995, he was a Visiting Professor with Stanford University. From 1996 to 2010, he was the Provost of Bilkent University, where he is currently the Rector. From 2004 and 2011, he served as a member of the Science Board of TUBITAK. His current research interests include microwave electronics and micromachined sensors. He received the Science Award of the Turkish Scientific Research Council (TUBITAK) in 1994. He has been a member of the Turkish Academy of Sciences since 1997.

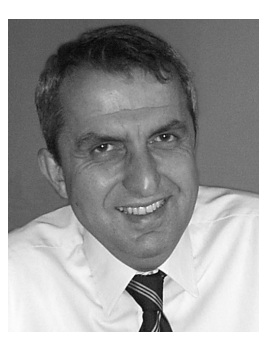

Hayrettin Köymen received the B.Sc. and M.Sc. degrees in electrical engineering from Middle East Technical University (METU), Ankara, Turkey, in 1973 and 1976, respectively, and the Ph.D. degree in electrical engineering from Birmingham University, Birmingham, U.K., in 1979. From 1979 to 1990 , he was with the Department of Marine Sciences, METU, Mersin, Turkey, and with the Department of Electrical Engineering, METU, Ankara. In 1990, he joined the Faculty of Bilkent University, Ankara, where he is currently a Professor with the Department of Electrical and Electronics Engineering. His research interests include underwater and airborne acoustic and ultrasonic transducer design, underwater acoustics, underwater and airborne acoustic systems, acoustic microscopy, ultrasonic NDT, biomedical instrumentation, mobile communications, and spectrum management. He is an IET Fellow. 